\title{
Effect of thermal annealing on sensing properties of optical fiber sensors coated with indium tin oxide nano-overlays
}

\author{
Bartosz Michalak, ${ }^{1,2 *}$, Petr Sezemsky, ${ }^{3}$ Vitezslav Stranak, ${ }^{3}$ and Mateusz Śmietana ${ }^{1}$ \\ ${ }^{1}$ Warsaw University of Technology, Institute of Microelectronics and Optoelectronics, \\ Koszykowa 75, 00-662, Warsaw Poland, \\ ${ }^{2}$ Warsaw University of Technology, Centre for Advanced Materials and Technologies CEZAMAT, \\ Poleczki 19, 02-822, Warsaw, Poland, \\ ${ }^{3}$ University of South Bohemia, Institute of Physics, Branisovska 1760, 37005 Ceske Budejovice, Czech Republic
}

Received May 17, 2020; accepted June 30, 2020; published June 30, 2020

\begin{abstract}
In this work we discuss an effect of thermal annealing on optical and electrical properties of indium tin oxide (ITO) thin films deposited on a short section of the multimode polymer-clad silica (PCS) optical fiber core. ITO films were deposited using a different configuration of high-power impulse magnetron sputtering (HiPIMS) and radio frequency magnetron sputtering (RF MS) cathodes. Due to tuned ITO film thickness it was possible to observe for these structures a lossy-mode resonance (LMR) and trace changes in the properties of films. Electrical resistance of the ITO overlays was also measured. Both optical and electrical measurements were repeated after annealing at $200^{\circ} \mathrm{C}$ in a tube furnace and nitrogen atmosphere. The measurements have shown that thermal annealing changes an optical response to the external refractive index (RI) of the fiber sensor and also changes the ITO layer resistivity. As an effect of thermal annealing, we observed a shift of the LMR towards longer wavelengths. In addition, as a result of annealing, the resistivity of the ITO layer was reduced.
\end{abstract}

In rising need for body fluid analysis, optical fiber sensors are considered as a suitable solution [1]. Systems based on optical fibers are modular and can be connected in series, which allows for simultaneous measurements of several parameters. Due to low fabrication cost, they can be disposed after contact with a patient's body fluids and replaced with new ones. To increase the reliability of measurements, an optical readout from sensors needs to be verified, most likely by the same setup but different sensing mechanism. One of the solutions is simultaneous optical and electrochemical analysis.

An optical effect employed in this work is lossy-mode resonance (LMR). It occurs when optical properties of the fiber core, deposited thin overlay and surrounding liquid are in a certain relation [2]. This relation is fulfilled when tuned- in- properties indium tin oxide (ITO) is deposited as an overlay. Tracing LMR also makes quick measurement of the refractive index (RI) of surrounding liquid possible. Due to high RI sensitivity, ITO-coated optical fiber structures can be used to measure, for example, the composition of a solution, and also the growth of a biological film on the sensor's surface [3]. Thanks to electrochemical activity of ITO, it can be also used for LMR simultaneous electrochemical

*E-mail: b.michalak@cezamat.eu measurement, which makes the cross-verification of results possible [1]. However, there is a need for postdeposition optimization of ITO properties for these simultaneous measurements, e.g., towards the reduction in resistivity of the overlays. As one of the postprocessing solutions can be considered thermal annealing.

In this work we discuss the effect of thermal annealing on optical and electrical properties of ITO overlays deposited, by using different magnetron sputtering configurations, on a short section of the multimode polymer-clad silica (PCS) optical fiber core. The annealing effect is analysed by comparing the optical response of the sensor, in particular when submerged in liquids of different RI, as well as by comparing the resistivity of the ITO-coated section.

The sensors were based on PCS optical fiber $(150 \mathrm{~mm}$ in length, $\varnothing=400 \mu \mathrm{m}$ fused silica core) where the central section had been chemically exposed to methylene chloride and then some of the cladding was mechanically removed to a length of $25 \mathrm{~mm}$. Next, ITO overlays were deposited on the exposed core section using a different configuration of high-power impulse magnetron sputtering (HiPIMS) and radio frequency magnetron sputtering (RF MS) cathodes [4]. HiPIMS provides high density plasma with pulses of high energy output which shortens the PCS samples exposure time to high temperature during the deposition process. The introduction of RF MS decreases the process time even further by speeding up the development of HiPIMS pulses and reducing the working pressure [5].

One run of deposition was done on three fiber samples called as A, B and C, installed in a metal frame that was placed on a rotating stage in the process chamber, and after a half of the process time the frame was flipped to the other side. At the top and on the side of the substrate, sputtering targets were installed in the process chamber (Fig. 1) in a different configuration of cathodes. The configuration for the sample series is summarized in Table 1. It was done to find the most suitable deposition process parameters. 
Optical transmission of the ITO-LMR samples was measured using an Ocean Optics USB4000 spectrometer and Ocean Optics HL-2000 white light source. To identify RI sensitivity, the samples were submerged in liquids with RI in the range $n_{D}=1.334 \div 1.466$. The $n_{D}$ of the liquid was measured using a Rudolph's Digital J57 refractometer. Obtained transmission spectra were normalized using the data set for a sample with no overlay measured in air.

Electrical resistance of the ITO layers was also measured on a $25 \mathrm{~mm}$ length of the sensor area using a digital multimeter.

After both optical and electrical measurements, the samples were annealed at $200^{\circ} \mathrm{C}$ in a tube furnace in a nitrogen atmosphere for 2 hours. Next, the measurements were repeated. In particular, we analysed the LMR shift observed in the spectrum as an effect of thermal annealing.

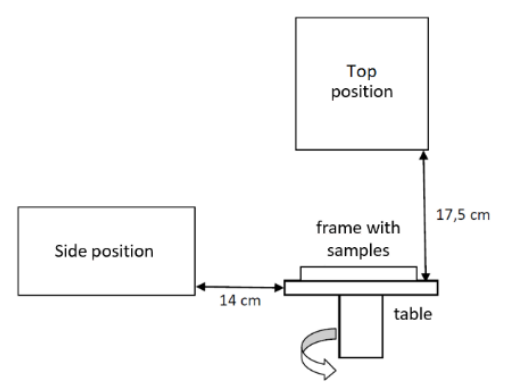

Fig. 1. Schematic representation of elements in the process chamber.

Table 1. Magnetron sputtering configurations used in the experiment.

\begin{tabular}{|c|c|c|c|}
\hline & 01 & 02 & 03 \\
\hline & RF MS & & HiPIMS \\
\hline Top position & $\begin{array}{l}3 \text { " ITO } \\
\text { target }\end{array}$ & - & $\begin{array}{c}2 \text { " ITO } \\
\text { target }\end{array}$ \\
\hline & HiPIMS & RF MS & RF MS \\
\hline Side position & $\begin{array}{l}2 " \text { ITO } \\
\text { target }\end{array}$ & $\begin{array}{l}3 \text { " ITO } \\
\text { target }\end{array}$ & $\begin{array}{l}\text { 3" ITO } \\
\text { target }\end{array}$ \\
\hline $\begin{array}{c}\text { Pressure } \\
{[\mathrm{Pa}]}\end{array}$ & 0.7 & 0.5 & 0.5 \\
\hline $\begin{array}{c}\text { Argon flow } \\
{[\mathrm{sccm}]}\end{array}$ & 15 & 15 & 15 \\
\hline Time [min] & 40 & 120 & 48 \\
\hline
\end{tabular}

Analysis of the obtained transmission spectra confirms that the position of the sputtered target led to a different thickness of overlays. The number of resonances, shown by black arrows (Fig. 2), indicates the deposited layer thickness - the more resonances, the thicker is the overlay [6]. When RF MS was at the top position and HiPIMS was on the side position, the configuration and $40 \mathrm{~min}$ long process led to the deposition of relatively thin overlays - two main resonances can be seen (Fig. 2a). When RF MS was only on the side, it led to an even thinner overlay deposition - only one resonance occurred, even after the process time was tripled (Fig. 2b). Using HiPIMS in the top position and RF MS on the side, thicker ITO overlays were achieved - four resonances can be seen (Fig. 2c).

The samples were submerged in liquids with RI in the range $n_{D}=1.334 \div 1.466$, where the brighter plots represent the lower RIs of a used liquid. In all cases, the observed resonances shifted towards longer wavelengths with external RI, shown by the orange arrow (Fig. 2).

Resistance measurements were made on three samples A, B and C, which were present during the same deposition process, expect for series 01 , which was not considered for resistivity measurement. We have observed differences in the resistance of samples made in the same process (Tab. 2). It may be caused by the spatial heterogeneity of plasma and its different interactions with the samples. Further analysis indicates that thicker layers show lower resistivity, while thermal annealing lowers it even more. Lower resistance helps in conducting efficient electrochemical measurements, and for this reason the contribution of HiPIMS is preferable.

Thermal annealing also affects optical transmission of optical fiber samples, the LMR shift towards longer wavelengths (Fig. 3). We can still observe the LMR shift towards longer wavelengths with an increase of RI. Due to the thermal annealing of optical fiber structure, the RI sensitivity decreased for all cases (Tab. 3.). This is an effect of ordering the crystallographic structure of the ITO layer, which leads to an increase of ITO overlay RI [7]. It is an undesirable effect from an optical sensing point of view. The effect of annealing is less pronounced for samples received with the HiPIMS contribution due to higher energy already delivered to sputtered particles in this process, compared to RF MS applied solely.
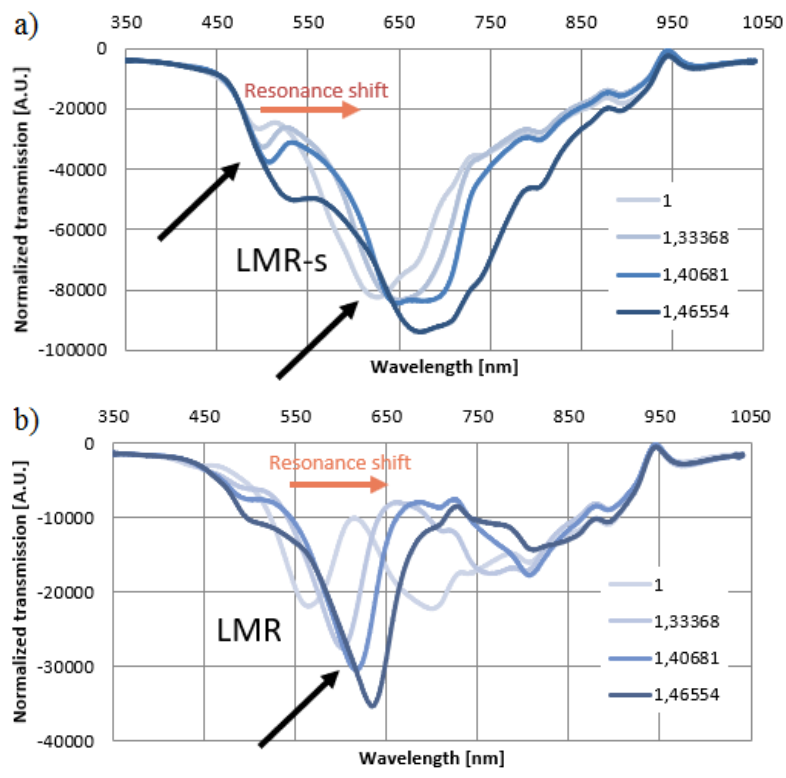

(C) 2020 Photonics Society of Poland 


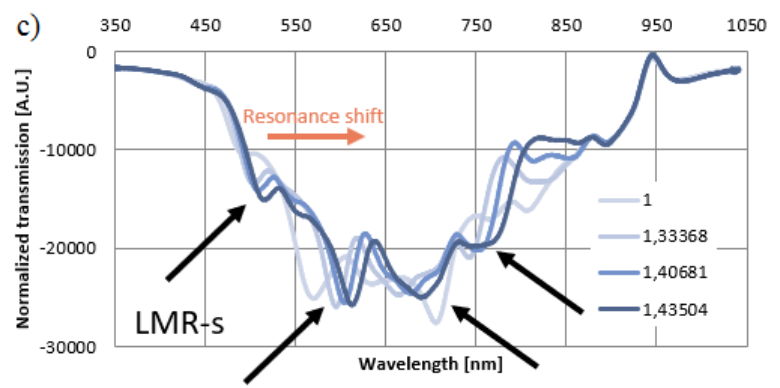

Fig. 2. Optical transmission spectra of a) 01, b) 02, c) 03, sample series and the LMR shift before thermal annealing when immersed in liquids of different RI.

Table 2. Resistance of three samples A, B and C from 02 and 03 series before and after annealing.

\begin{tabular}{|c|c|c|}
\hline & $\begin{array}{c}\text { Resistance before } \\
\text { annealing }[\Omega]\end{array}$ & $\begin{array}{c}\text { Resistance after } \\
\text { annealing }[\Omega]\end{array}$ \\
\hline 02A & - & 157000 \\
\hline 02B & - & 250000 \\
\hline 02C & - & 270000 \\
\hline 03A & 360 & 250 \\
\hline 03B & 320 & 300 \\
\hline 03C & 500 & 270 \\
\hline
\end{tabular}
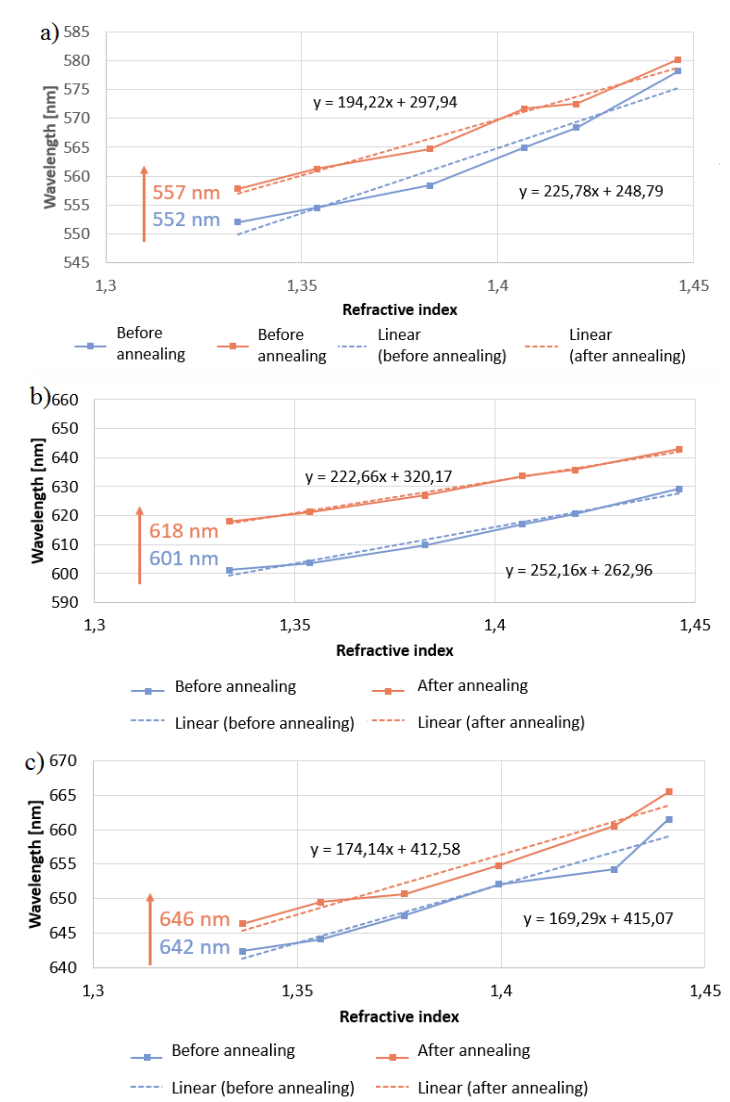

Fig. 3. Resonance shift of a) 01 , b) 02 , c) 03 sample series with linear approximation before and after thermal annealing when immersed in liquids of different RI.
Table 3. RI sensitivity for 01,02 and 03 series before and after annealing.

\begin{tabular}{|c|c|c|}
\hline & $\begin{array}{c}\text { Sensitivity before } \\
\text { annealing } \\
{[\mathrm{nm} / \text { RIU] }}\end{array}$ & $\begin{array}{c}\text { Sensitivity after } \\
\text { annealing } \\
\text { [nm/RIU] }\end{array}$ \\
\hline 01 & 232.05 & 199.22 \\
\hline 02 & 249.49 & 221.55 \\
\hline 03 & 183.30 & 183.11 \\
\hline
\end{tabular}

In conclusions, obtained data indicates that ITO films obtained with a contribution of HiPIMS are more resistant to thermal annealing than those deposited using RF MS only. Optical fibers with an ITO layer are sensitive to changes in external RI and respond as an LMR shift towards longer wavelengths. When LMR sensors are considered, thermal annealing changes the optical response of the ITO-coated sensor and, in general, lowers its optical sensitivity. Observed was an LMRs shift with annealing towards longer wavelengths. However, annealing also decreased electrical resistance of the deposited ITO. Understanding both effects can lead to finding optimal ITO deposition parameters and also optimal annealing temperature for optical fiber sensors used in simultaneous optical and electrochemical

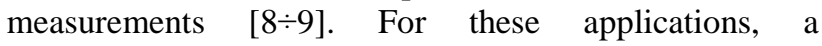
contribution of HiPIMS is expected.

The authors gratefully acknowledge the support for this work from the National Science Centre of Poland within the research project 2014/14/E/ST7/00104 and The National Centre for Research and Development of Poland within the research project TECHMATSTRATEG1/347324/12/NCBR/2017

\section{References}

[1] M. Sobaszek et al., Proc. SPIE 10323, 103234W (2017).

[2] I. Del Villar et al., J. Opt. 12(9), 95503 (2010).

[3] M. Śmietana et al., Biosensors and Bioelectronics 154, 112050 (2020).

[4] P. Niedziałkowski et al., Sensors and Actuators B: Chemical 301(12), 1 (2019).

[5] V. Stranak et al., Surface \& Coatings Technology 206(11-12), 2801 (2012).

[6] I. Del Villar et al., Appl. Opt. 51(19), 4298 (2012).

[7] W.F. Wu, B.S. Chiou, Appl. Surf. Sci. 68(4), 497 (1993).

[8] M. Smietana et al., J. Light. Technol. 36(4), 954 (2018).

[9] M. Śmietana et al., Electroanalysis 31(2), 398 (2019). 